This item was submitted to Loughborough's Research Repository by the author.

Items in Figshare are protected by copyright, with all rights reserved, unless otherwise indicated.

\title{
Bayesian estimation of a periodically-releasing biochemical source using
} sensor networks

PLEASE CITE THE PUBLISHED VERSION

https://doi.org/10.1109/CONTROL.2018.8516751

PUBLISHER

(C) IEEE

VERSION

AM (Accepted Manuscript)

LICENCE

CC BY-NC-ND 4.0

\section{REPOSITORY RECORD}

Hu, Liang [Computer Science], Jinya Su, Michael Hutchinson, Cunjia Liu, and Wen-Hua Chen. 2018. "Bayesian Estimation of a Periodically-releasing Biochemical Source Using Sensor Networks". figshare. https://hdl.handle.net/2134/33824. 


\section{Bayesian Estimation of A Periodically-Releasing Biochemical Source Using Sensor Networks}

\author{
Liang $\mathrm{Hu}$ \\ School of Computer Science and Informatics \\ De Montfort University \\ Leicester, UK \\ liang.hu@dmu.ac.uk
}

\author{
Jinya Su, Michael Hutchinson, Cunjia Liu, Wen-hua Chen \\ Department of Aeronautical and Automotive Engineering \\ Loughborough University \\ Loughborough, UK \\ \{J.Su2, M.Hutchinson2, C.Liu5, W.Chen\}@lboro.ac.uk
}

\begin{abstract}
This paper develops a Bayesian estimation method to estimate source parameters of a biochemical source using a network of sensors. Based on existing models of continuous and instantaneous releases, a model of discrete and periodic releases is proposed, which has extra parameters such as the time interval between two successive releases. Different from existing source term estimation methods, based on the sensor characteristic of chemical sensors, the zero readings of sensors are exploited in our algorithm where the zero readings may be caused by the concentration being below the threshold of the sensors. Two types of Bayesian inference algorithms for key parameters of the sources are developed and their particle filtering implementation is discussed. The efficiency of the proposed algorithms for periodic release is demonstrated and verified by simulation where the algorithm with the exploitation of the zero readings significantly outperforms that without.
\end{abstract}

Index Terms-Source-term estimation, Bayesian estimation, Atmospheric dispersion model, Sensor networks

\section{INTRODUCTION}

Toxic or harmful materials released from biochemical sources, which typically spread widely via winds in the atmosphere, pose serious threats to both natural environment and human lives. Motivated by the growing concern of potential risks of biochemical accidents or attacks in the modern society, recently increasing research attentions have been attracted to early detection and rapid localisation of sources of biochemical releases [1], [2].

To estimate the source parameters from the contaminant detected in an area of interest, a set of measurement data on the concentration of the contaminant in the atmosphere and a relevant atmospheric transport and dispersion (ATD) model of the contaminant are needed. To obtain the measurement data, typically a network of static or mobile sensors are distributed spatially at different locations to measure the concentration of biochemical substance in the atmosphere [3], [4], [9]. On the other hand, ATD models describe how the biochemical materials spread in different atmospheric conditions [5]. Based on an assumed ATD model and real-time measurement data from sensors, various methods have been proposed to estimate the parameters of the source releasing biochemical materials,

This work was supported by the Engineering and Physical Sciences Research Council (EPSRC) Grant number EP/K014307/1 and the MOD University Defence Research Collaboration in Signal Processing. of which the optimization-based technique and the Bayesian technique are two typical approaches as reviewed in [2]. Compared with the optimization-based techniques [6], [7], the Bayesian technique has the advantage that it quantifies the estimation uncertainty by providing a probability density distribution of the source-term parameter [8]. In this work, we try to develop a Bayesian estimation approach for the source term estimation (STE) problem using the measurment of a set of static sensors.

How to localise and estimate a biochemical source that releases discretely instead of continuously is our first research motivation. As discussed in [17], most of existing researches on source localisation are based on the assumption of continuously emitting sources. Such an assumption simplifies the problem as the classic Gaussian plume model applies well. However, the Gaussian plume model cannot be adapted to discretely releasing sources because, as a steady-state model, it cannot characterise the concentration distribution formed by a series of discretely-releasing events. To bridge the gap, we firstly develop an ATD model for the discretely-releasing source based on the Gaussian puff model of instantaneouslyrelease source [17]. Specifically, the essential parameters of the release starting time and the time interval between two successive releases are both embedded in the developed model.

The second research motivation comes for the phenomenon observed from experimental data of field tests on boichemical source localisation. As described in [11]-[13], it is found that a considerable number of sensors in the field tests do not report any reading even though no faults happen in these sensors. In practice, due to the intrinsic limits of sensors, a sensor will not be triggered to output a reading if the concentration level is less than the sensor's threshold. Under turbulent meteorological conditions, the concentration distribution of biochemical contaminant in the atmosphere varies greatly from location to location. As a result, the concentration at the locations far away from the puff centre is highly likely to be less than the threshold of the sensors. Traditionally, only the valid measurements received from the sensors are used to estimate the source-term parameters while all the "silent" sensors without readings are ignored. Actually, the non-existance of reading itself does provide useful information. That is, the concentrations at the locations of the "silent" sensors are less 
than the sensors' threshold. Such hidden information, once being exploited appropriately through information fusion, may substantially improve estimation performance of the source parameters.

The phenomenon of non-existence of readings has been noticed and addressed in [18], [19], where a probabilistic model of the existence and non-existence (called detection/nondetection in [18], [19]) event was assumed. In this paper, we will not follow such an assumption as the model parameters might be difficult or impossible to known in real-world applications. Instead, we formalise the observation that sensors will have no readings if and only if the concentration is below the threshold of the sensors.

In this paper, a particle filter based algorithm is proposed for the STE problem of the discretely-releasing source. First, the STE problem is formally defined based on our proposed Gaussian model for discrete releases. Then a particle filtering framework is adopted to estimate the source parameters. In particular, a customed importance weight updating step is put forward in the proposed particle filtering algorithm that takes into account of measurements of all sensors: both the ones with and the ones without readings. Following the formula of Bayes theorem, the hidden information of no reading from sensors are incorporated explicitly in the process of Bayesian inference. Simulations are presented to compare the performance of our proposed algorithm to the one that considers only sensors that report readings.

The remainder of the paper is organised as follows. In Section II, preliminary knowledges of ATD models and the measurement model are presented and the Gussian model for discrete releases is developed. The STE problem we investigate in this paper is formally presented in Section III. In Section IV, the conceptual solution is described followed by the sequential Bayesian algorithm used to implement the conceptual solution. An illustrative run and Monte Carlo simulations with other strategies are presented and discussed in Section V. The paper is finally concluded and the future research direction is put forward in Section VI.

\section{PREliminary Models}

Depending on the release patterns of the source, e.g. continuous or instantaneous, different ATD models are proposed to describe the concentration distributions formed by the emitted materials in the atmosphere. In this paper, we aim to estimate source term parameters of discrete releases. To the end, we first briefly introduce two established Gaussian models and then extend them to modelling the atmospheric dispersion of discrete releases. Based upon the proposed model, we define the STE problem of discrete releases formally.

\section{A. Gaussian plume and puff models}

Two classic Gaussian models are widely used for describing the ATD process. The Gaussian plume model applies well if a steady atmospheric dispersion is formed by continuous biochemical release, while the Gaussian puff model characterizes the dispersion process after an instantaneous release.
Gaussian plume model: this model is derived from the turbulent diffusion equation under the assumption of constant wind speed and a point source continuously releases with constant rate [5]. Define the downwind, crosswind and vertical directions as the $x$-axis $y$-axis, and $z$-axis, respectively. The concentration of the plume at location $P$ is given as follows:

$$
\begin{aligned}
C_{1}\left(x, y, z, \theta_{1}\right) & =\frac{q_{s}}{2 \pi \bar{u} \sigma_{y} \sigma_{z}} \exp \left(\frac{-\left(y-y_{s}\right)^{2}}{2 \sigma_{y}^{2}}\right) \\
& \times\left[\exp \left(\frac{-\left(z-z_{s}\right)^{2}}{2 \sigma_{z}^{2}}\right)+\exp \left(\frac{-\left(z+z_{s}\right)^{2}}{2 \sigma_{z}^{2}}\right)\right]
\end{aligned}
$$

where $(x, y, z)$ is the coordinate of the location $P$ and $\theta_{1}$ is the source-term parameter vector, i.e., $\theta_{1}=\left[\begin{array}{llll}q_{s} & x_{s} & y_{s} & z_{s}\end{array}\right]^{T}$, and $q_{s}$ is the release rate, $\left(x_{s}, y_{s}, z_{s}\right)$ is the coordinate of the source location, and $\bar{u}$ is the mean wind speed. The dispersion parameters $\sigma_{y}$ and $\sigma_{z}$ are the standard deviations of the Gaussian model in the crosswind and vertical directions, defined as a function of downwind distance:

$$
\left\{\begin{array}{l}
\sigma_{y}=a\left(x-x_{s}\right)^{b} \\
\sigma_{z}=c\left(x-x_{s}\right)^{d}
\end{array}\right.
$$

where the variables $(a ; b ; c ; d)$ are a function of stability category, whose actual values are stochastic variables around the reference values given for a specific stability category [5]. for example at Pascal stability category $\mathrm{C}$, the reference values are $a=0.66 ; b=0.81 ; c=0.17$ and $d=1$.

Gaussian puff model: this model is adopted to characterise the ATD of an instantaneous rather than continuous release [5], [17]. The model is given as follows:

$$
\begin{aligned}
& C_{2}\left(x, y, z, \theta_{1}, t_{s}, t\right) \\
= & \frac{q_{s}}{(2 \pi)^{1.5} \sigma_{x} \sigma_{y} \sigma_{z}} \exp \left[-\frac{\left(x-x_{c}\right)^{2}}{2 \sigma_{x}^{2}}-\frac{\left(y-y_{c}\right)^{2}}{2 \sigma_{y}^{2}}\right] \\
& \times\left[\exp \left(\frac{-\left(z-z_{s}\right)^{2}}{2 \sigma_{z}^{2}}\right)+\exp \left(\frac{-\left(z-z_{s}\right)^{2}}{2 \sigma_{z}^{2}}\right)\right]
\end{aligned}
$$

where $q_{s}$ is the mass of instantaneous release, $(x, y, z)$ is the coordinate of the location $P$ and $\left(x_{s}, y_{s}, z_{s}\right)$ is the coordinate of the source location $P_{s}, \bar{u}$ is the mean wind speed, and $\sigma_{x}$, $\sigma_{y}$ and $\sigma_{z}$ are the dispersion coefficients. The coordinates of the centroid of the puff $\left(x_{c}, y_{c}\right)$ is moved by the wind over time according to the following equations:

$$
\left\{\begin{array}{l}
x_{c}=x_{s}+\bar{u}_{x}\left(t-t_{s}\right) \\
y_{c}=y_{s}+\bar{u}_{y}\left(t-t_{s}\right)
\end{array}\right.
$$

where $t_{s}$ is the time of the instantaneous emission, $\bar{u}_{x}$ and $\bar{u}_{y}$ are the wind components at the $x$-axis and $y$-axis. The dispersion parameters $\sigma_{x}, \sigma_{y}$ and $\sigma_{z}$ have the same defination as in the Gassian plume model except that $\sigma_{x}=\sigma_{y}$.

\section{B. Gaussian model for discretely periodic release}

Since the ATD process of materials released periodically at discrete times cannot be described by the Gaussian plume model (1) or the Gussian puff model (3), we first extend the model (3) to describe the case of periodic release at discrete 
times. Assume that the source starts the first release at time instant $t_{s}$, and then emits regularly at a fixed time interval $\tau$. The extended Gaussian model for discrete releases we propose is given as follows:

$$
\begin{array}{r}
C(x, y, z, \theta, t)=: C\left(x, y, z, \theta_{1}, t_{s}, \tau, t\right) \\
=\sum_{l=0}^{\left\lceil\left(t-t_{s}\right) / \tau\right\rceil-1} C_{2}\left(x, y, z, t_{s}+l \tau, t\right)
\end{array}
$$

where $C_{2}(\cdot)$ is defined in (3), and $\left\lceil\left(t-t_{s}\right) / \tau\right\rceil$ represents the number of release times between the first release time instant $t_{s}$ and the current time instant $t$. The parameter vector $\theta$ consists of 6 parameter variables, that is:

$$
\theta=\left[\begin{array}{llllll}
q_{s} & x_{s} & y_{s} & z_{s} & t_{s} & \tau
\end{array}\right]^{T} .
$$

Note that the instantaneous model (3) is a special case of the discrete model (5) by setting $\tau=+\infty$. On the other hand, the discrete model (5) reduces to the steady Gaussian plume model if the release interval $\tau \rightarrow 0$. From this perspective, the proposed discrete model bridges the two existing Gaussian models in a unified framework.

The discretely periodic release model can be further extended to model discrete (not necessarily periodic) releases by relaxing the assumption that the time interval between each two consecutive releases is constant. In this sense, the proposed model is a starting point for researches on the STE problem with discrete releases.

\section{Measurement model}

Various sensors are capable of measuring the concentration of biochemical substance in the atmosphere, but all sensors suffer measurement noises, which is commonly characterised by a Gaussian white noise. Ideally, the measurement model of a sensor at location $(x, y, z)$ for discrete releases is given as follows:

$$
z(t)=C(x, y, z, \theta, t)+\omega(t)
$$

where $C(\cdot)$ takes the form of $(5)$, and $\omega(t)$ is a zero-mean Gaussian white noise with covariance $W$.

In practice, due to the intrinsic limits of sensors, a sensor will not output reading if the concentration level is less than the sensor's threshold. A simulation example run of discrete release is shown in Fig. 1, where the red star denotes the static source position, 36 sensors measure the concentrations and the colour map represents the concentration at positions in the $x$ and $y$ frames. It is found that quite a few sensors have no output readings. This kind of phenomenon is widely encountered when measuring the biochemical concentration in atmospheric environment.

To take into account the non-existance of readings due to sensor thresholds, we propose the following measurement model:

$$
z(k)=\left\{\begin{array}{cl}
z(k), & \text { if } z(k)>\delta \\
\emptyset, & \text { if } z(k) \leq \delta
\end{array}\right.
$$

where $z(k)$ is the ideal measurement defined in (6), and $\delta$ is the threshold of the sensor. If the measurement $z(k)$ is not

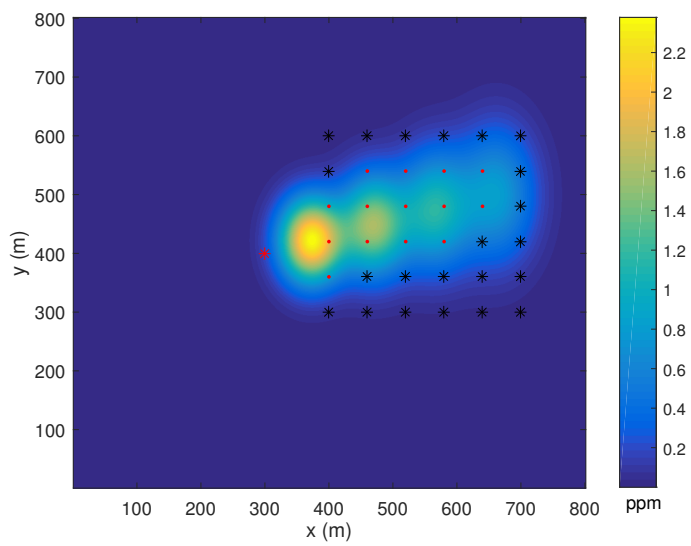

Fig. 1. Example plot of the concentration from a discretely-releasing source. The red star indicates the origin of the source, the red dots indicate sensors with readings while the black stars sensors without readings. The colour map denotes the concentration at the correspondence position, generated from a discrete release.

bigger that the threshold, then the sensor will not be triggered to output a reading.

\section{PROBLEM FORMULATION}

In this paper, a point source is assumed to release biochemical substance regularly at discrete times. Suppose that $n$ sensors with the same measurement threshold $\delta$ are placed in arbitrary but known locations to measure the contaminant concentration. The sensors send the measurement to a fusion centre with a sampling interval $T$. Define the time instant that the first time at least one sensor measures a non-zero concentration as the time instant 0 . The concentration measurements from all $n$ sensors at time instant $k T$ is denoted as $z(k)=\left[z_{1}(k), \ldots, z_{n}(k)\right]^{T}$.

Denote the location of the $i$ th sensor by $\left(x_{i}, y_{i}, z_{i}\right)$ and the measurement from the $i$ th sensor by $z_{i}(t)$. Define the sets

$$
\begin{aligned}
& Z_{1}(k)=\left\{z_{i}(k) \mid z_{i}(k)=\emptyset\right\} \\
& Z_{2}(k)=\left\{z_{i}(k) \mid z_{i}(k) \neq \emptyset\right\}
\end{aligned}
$$

A cumulative set of all temporal measurements available up to time $k T$ is denoted as $\mathbf{Z}(\mathbf{k}):=\{z(j), j=0, \ldots, k\}$. More specifically, define the cumulative set of non-existance of readings measurements $\mathbf{Z}_{\mathbf{1}}(\mathbf{k}):=\left\{z_{i}(j) \mid z_{i}(j)=\emptyset, i=\right.$ $1, \ldots, n, j=0, \ldots, k\}$ and that of the real measurements $\mathbf{Z}_{\mathbf{2}}(\mathbf{k}):=\left\{z_{i}(j) \mid z_{i}(j) \neq \emptyset, i=1, \ldots, n, j=0, \ldots, k\right\}$.

To exploit the information of nonexistance of sensor readings in Bayesian estimation, the conditional probability of the event of a sensor without reading is written explicitly as follows:

$$
\begin{array}{r}
\operatorname{Pr}\left(z_{i}(k) \in Z_{1}(k) \mid \theta\right)=\operatorname{Pr}\left(z_{i}(k)-\delta \leq 0\right) \\
=\Phi\left(\frac{\delta-C\left(x_{i}, y_{i}, z_{i}, \theta, k T\right)}{W^{\frac{1}{2}}}\right)
\end{array}
$$

where $\Phi(x)$ is the normalized form of the cumulative normal distribution function $\Phi(x)=\frac{1}{\sqrt{2 \pi}} \int_{0}^{x} e^{-t^{2} / 2} \mathrm{~d} t$. 
Given the measurement sequence $\mathbf{Z}(\mathbf{k})$, the prior information $\pi(\theta)$ of the source parameters and the knowledge of the measurement model (7), we aim to obtain the probabilistic distribution $p(\theta \mid \mathbf{Z}(\mathbf{k}))$ of the source parameter $\theta$ at each time step $k T$ in a sequential approach.

\section{BAYESIAN ESTIMATION FOR STE}

In this section, we present the Bayesian approach to the STE problem of discretely-releasing source. To continuously update the estimation based on new measurement data received in sequences, the proposed approach is performed in a sequential manner.

\section{A. Conceptual solution}

The STE problem is formulated in a Bayesian framework that the source parameters are estimated using Bayes theorem when new measurement data is obtained at each time step. The posterior probability density of $\theta$ is derived in the following recursive form:

$$
\begin{aligned}
p(\theta \mid \mathbf{Z}(\mathbf{k}))= & \frac{p\left(Z_{1}(k), Z_{2}(k) \mid \theta, \mathbf{Z}(\mathbf{k}-\mathbf{1})\right) p(\theta \mid \mathbf{Z}(\mathbf{k}-\mathbf{1}))}{p\left(Z_{1}(k), Z_{2}(k) \mid \mathbf{Z}(\mathbf{k}-\mathbf{1})\right)} \\
\propto & \operatorname{Pr}\left(Z_{1}(k) \mid \theta\right) p\left(Z_{2}(k) \mid \theta\right) p(\theta \mid \mathbf{Z}(\mathbf{k}-\mathbf{1})) \\
\propto & \operatorname{Pr}\left(Z_{1}(k) \mid \theta\right) \operatorname{Pr}\left(Z_{1}(k-1) \mid \theta\right) p\left(Z_{2}(k) \mid \theta\right) \\
& \times p\left(Z_{2}(k-1) \mid \theta\right) p(\theta \mid \mathbf{Z}(\mathbf{k}-\mathbf{2})) \\
\propto & \pi(\theta) \prod_{j=0}^{k} \operatorname{Pr}\left(Z_{1}(j) \mid \theta\right) p\left(Z_{2}(j) \mid \theta\right)
\end{aligned}
$$

where $p(\theta \mid \mathbf{Z}(\mathbf{j}))$ refers to the posterior probability density of $\theta$ given the measurement set $\mathbf{Z}(\mathbf{j}), p\left(Z_{2}(j) \mid \theta\right)$ is the likelihood function of measurement $Z_{2}(j), \operatorname{Pr}\left(Z_{1}(j) \mid \theta\right)$ is the conditional probability of non-existance of sensor reading $Z_{1}(j)$ given the parameter $\theta$, and $\pi(\theta)$ is the prior probability density of the parameter $\theta$.

Due to the independence of the observation noises over different sensors, the likelihood $p\left(Z_{2}(j) \mid \theta\right)$ can be written as:

$$
p\left(Z_{2}(j) \mid \theta\right)=\prod_{i=1}^{n_{1}} \mathcal{N}\left(z_{i}-C\left(x_{i}, y_{i}, z_{i}, \theta, k T\right), W^{\frac{1}{2}}\right)
$$

where $n_{1}$ is the number of sensors without readings.

Note that not only the readings from sensors $Z_{2}(k)$, but also the non-existance of readings $Z_{1}(k)$ representing an extra information, is taken into account in the above Bayesian inference. Thus a more accurate estimate is expected to be obtained.

\section{B. Particle filter with non-existance of sensor readings}

In general, when a measurement model is nonlinear/nonGaussian, there does not exist analytically closed-from solution to the posterior probabilistic density [10], [14]. Instead numerically efficient approaches such as sequential Monte Carlo methods are used. In the following subsection, we will propose a sequential Monte Carlo method for STE based on the basic sequential importance-resampling structure of particle filter.

In this framework the posterior probabilistic density is represented by a set of random samples $\left\{\omega_{k}^{j}, \theta_{k}^{j}\right\}_{j=1}^{N}$ as

$$
p(\theta(k) \mid \mathbf{Z}(\mathbf{k})) \approx \sum_{j=1}^{N} \omega_{k}^{j} \Delta\left(\theta(k)-\theta_{k}^{j}\right)
$$

where $N$ is the number of the samples (particles), $\omega_{k}^{j}$ is the normalised importance weight of particle $j, j=1, \ldots, N$, $\Delta(\cdot)$ is the Dirac function. Each particle represents the source parameter vector. Furthermore, the characteristics of interest for source parameters can be approximated by the particles. For example, the mean of source parameters $\bar{\theta}(k)=$ $\sum_{j=1}^{N} \omega_{k}^{j} \theta_{k}^{j}$.

Before any measurement is received (at $k=0$ ), the particles are initialised and generated from the prior probabilistic density with equal initial weights $\omega_{0}^{j}=1 / N$. After receiving measurement data $z(k)(k=1,2, \ldots)$, the importance weight of the particles is updated according to

$$
\tilde{\omega}_{k}^{j}=\operatorname{Pr}\left(Z_{1}(k) \mid \theta_{k}^{j}\right) p\left(Z_{2}(k) \mid \theta_{k}^{j}\right) \omega_{k-1}^{j}
$$

where $\omega_{k-1}^{j}$ the normalized weight of particle $j$ at the previous time step $k-1, \operatorname{Pr}\left(Z_{1}(k) \mid \theta_{k}^{j}\right)$ is the probability of the nonexistance of readings given in (10), and $p\left(Z_{2}(k) \mid \theta_{k}^{j}\right)$ is the likelihood given in (12). In this step, the extra information that the concentration at the locations of the sensors without readings is less than the sensors' threshold is incorporated into the process of Bayesian inference. The weights are further normalized according to $\omega_{k}^{j}=\frac{\tilde{\omega}_{k}^{j}}{\sum_{j=1}^{N} \tilde{\omega}_{k}^{j}}$.

To prevent the degeneracy of the propagated particles, the particles are resampled using the system resampling method [15], and thus all weights of the particles equal to $1 / N$ after the resampling step.

Since the STE problem considered is essentilly a static parameter estimation problem, the variety of particles will decrease with steps as all particles will stay at the same locations by sampling from the stationary evolution $\theta(k)=\theta(k-1)$. As a result, the particles may converge to a single point very soon. To overcome the described deficiency, we adopt the regularisation techniques to improve the sample diversity [10], [16]. The particles are moved to new places according to the Gaussian Kernel density. The complete details of the proposed sequential Bayesian estimation method are described in Algorithm 1.

Compared with the Markov chain Monte Carlo (MCMC) based STE algorithms presented in [18], [19], the algorithm proposed in this paper works in a sequential approach and thus leads to less computation burden, which enables a fast and real-time application for STE.

\section{Simulationd And AnAlysis}

For briefness we only simulate the two-dimensional model on the horizontal plane (the $\mathrm{X}-\mathrm{Y}$ axis) though it is quite straightforward to extend to the three-dimensional one. The 


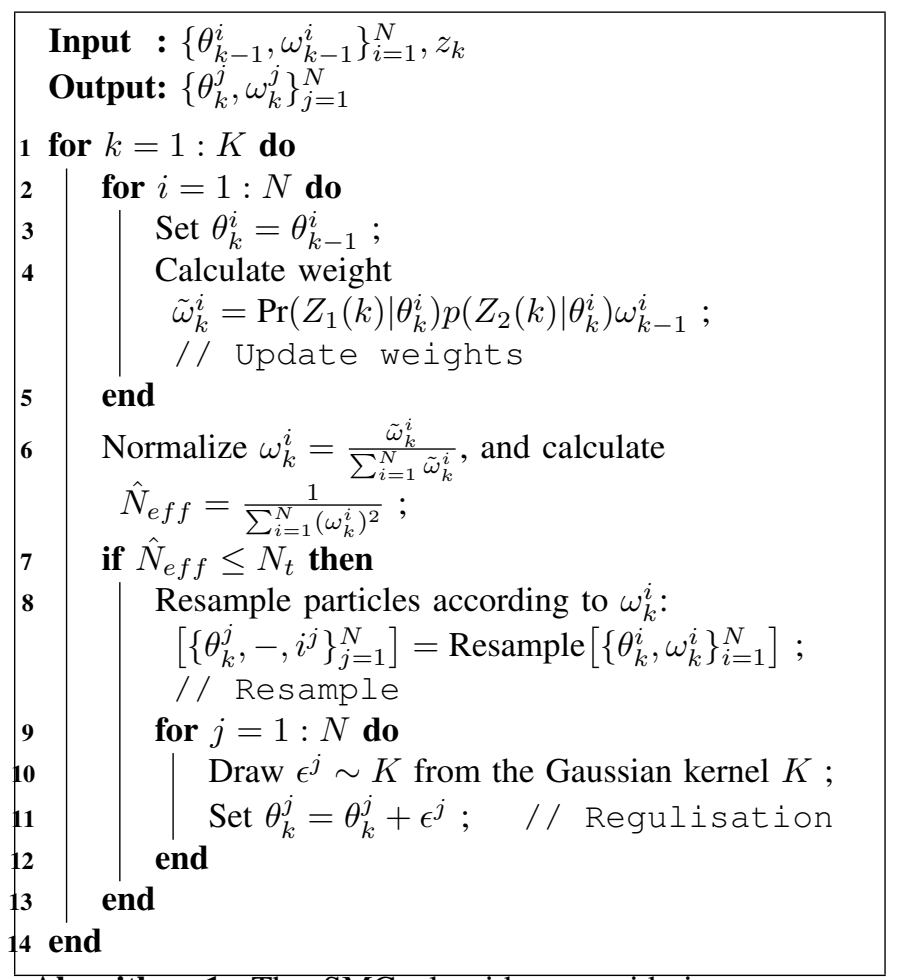

Algorithm 1: The SMC algorithm considering zeroreading

simulation is set up as follows. The rectangular area to be monitored with one biochemical source (indicated by the red star) is shown in Fig.1. The parameters of source are: its coordinates $x=300 \mathrm{~m}$ and $y=400 \mathrm{~m}$, the release mass $q=1200 \mathrm{~g}$, the time interval between each two consecutive releases $\tau=20 \mathrm{~s}$, and the release starting time $t_{s}=-300$ s. An array of 36 sensors are uniformly distributed in the square area where the bottom-left location is at $(400 \mathrm{~m}, 300 \mathrm{~m})$ and the top-right location is at $(700 \mathrm{~m}, 600 \mathrm{~m})$. All sensor are assumed to have the zero-mean Gaussian noise with covariance 0.01 . the threshold of the sensors is $1 \mathrm{~g} / \mathrm{m}(1 \mathrm{ppm})$ and the sampling time is $10 \mathrm{~s}$.

The atmospheric condition are assumed to fall into Pascal stability category $\mathrm{C}$, then the values of the dispersion parameters in (2) are $a=0.66 ; b=0.81 ; c=0.17$ and $d=1$. The wind has a speed of $4 \mathrm{~m} / \mathrm{s}$ and in the direction of $15^{0}$ from the x-axis. A population of 5000 particles is used in the simulation. The prior of all paramters are assumed to be uniform distributions: $q_{s} \sim[0,2000], \tau \sim[0,100]$, $t_{s} \sim[-600,0], x \sim[0,500]$, and $y \sim[0,500]$.

We have run two comparative simulations under the same setup: our proposed method, labelled as method I thereafter, considers the readings of all the sensors (including the ones without readings) while the other one, labelled as method II thereafter, considers only the readings reported by the sensors (excluding the sensor without readings). Figs. 2-6 show an illustrative run where the posterior estimates of the source parameters at different sampling instants are shown in the histograms and the red line is the true value of the parameters. It can be found from the simulation results that both STE methods produce more accurate estimation as more new measurement data are obtained with times. However, our proposed method (method I) taking into all sensor output yields better estimation performance. Such a conclusion is further validated by comparison of the root-mean-square error (RMSE) results after 100 Monte Carlo simulations as shown in Table I.

TABLE I

THE RMSE COMPARISION OF THE TWO METHODS

\begin{tabular}{l|c|r}
\hline RMSE & Method I & Method II \\
\hline Release rate (g) & 114.43 & 559.24 \\
Release interval (s) & 3.47 & 10.26 \\
Release start time (s) & 15.88 & 31.68 \\
X coordinate (m) & 69.67 & 71.39 \\
Y coordinate (m) & 15.54 & 19.17 \\
\hline
\end{tabular}

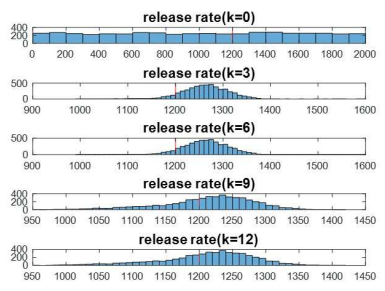

(a) method I

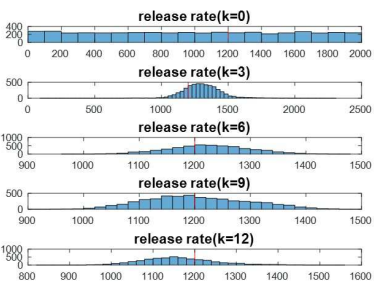

(b) method II
Fig. 2. The estimate of the release rate

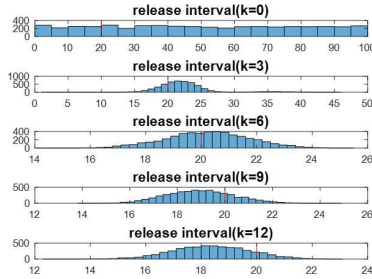

(a) method I

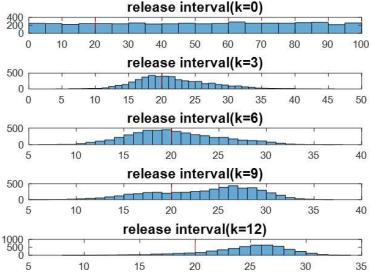

(b) method II
Fig. 3. The estimate of the release interval

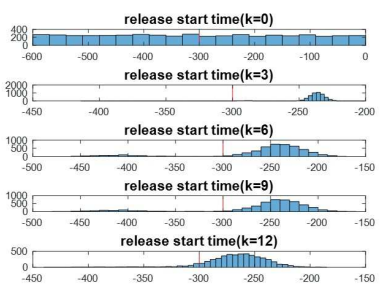

(a) method I

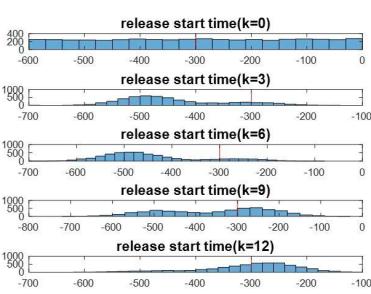

(b) method II
Fig. 4. The estimate of the release starting time

\section{CONCLUSION}

In this paper, a sequential Bayesian estimation method has been proposed to estimate the source term parameters of 


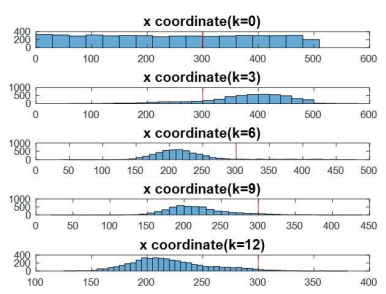

(a) method I

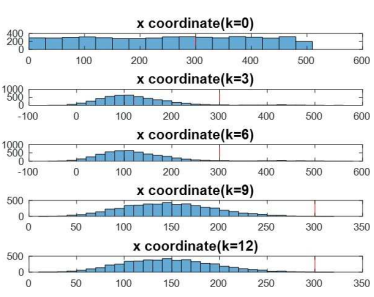

(b) method II
Fig. 5. The estimate of the $\mathrm{x}$ coordinate of the source location

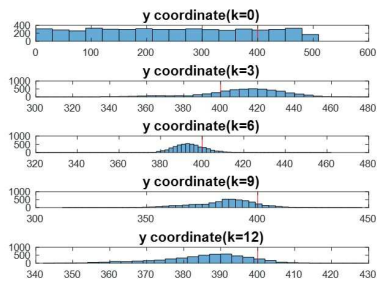

(a) method I

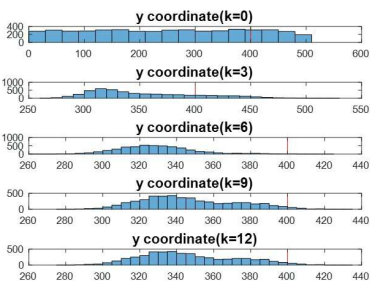

(b) method II
Fig. 6. The estimate of the y coordinate of the source location

discrete releases of biochemical material into the atmosphere. A practical atmospheric transport and dispersion model of discrete releases has been first proposed based on existing model of instantaneous release and that of continuous releases. To make the best of all sensors that measure the concentrations of the biochemical materials in atmosphere, the sensors that are not triggered to report readings are characterised via an explicit probabilistic discription. Furthermore, such a characterisation is embedded in the proposed particle filtering algorithm for source term estimation. Simulations demonstrate the effectiveness of our proposed method in estimating the source term parameters of discrete releases.

In this paper, it is assumed as a priori that the releases occur regularly in discrete times. In practical applications, it is quite possible that it is not known beforehand whether the release is continuous, discrete or instantaneous. Instead the release model need to be estimated in real time. One of our future research direction is to develop an estimation method that is able to identify the release model and to estimate the source parameters simultaneously using the multiple model based particle filter techniques.

\section{REFERENCES}

[1] Wang, Y., Huang, H., Huang, L. and Ristic, B., 2017. Evaluation of Bayesian source estimation methods with Prairie Grass observations and Gaussian plume model: A comparison of likelihood functions and distance measures. Atmospheric Environment, 152, pp.519-530.

[2] Hutchinson, M., Oh, H. and Chen, W.H., 2017. A review of source term estimation methods for atmospheric dispersion events using static or mobile sensors. Information Fusion, 36, pp.130-148.

[3] Hutchinson, M., Oh, H. and Chen, W.H., 2017. Adaptive Bayesian sensor motion planning for hazardous source term reconstruction. IFAC World Congress, France.

[4] Gunatilaka, A., Ristic, B., Skvortsov, A. and Morelande, M., 2008. Parameter estimation of a continuous chemical plume source. In: 11th International Conference on Information Fusion, pp. 1-8.
[5] Panofsky, H.A. and Dutton, J.A., 1984. Atmospheric turbulence: models and methods for engineering applications (No. 04; QC880. 4. T8, P3.). Wiley.

[6] Madankan, R., Singla, P. and Singh, T., 2015. Parameter estimation of atmospheric release incidents using maximal information collection. In Dynamic Data-Driven Environmental Systems Science (pp. 310-321). Springer, Cham.

[7] Singh, S.K. and Rani, R., 2014. A least-squares inversion technique for identification of a point release: application to fusion field trials 2007, Atmos. Environ. 92, pp. 104117.

[8] Ristic, B., Gunatilaka, A. and Gailis, R., 2014. Achievable accuracy in parameter estimation of a Gaussian plume dispersion model, in: 2014 IEEE Workshop on Statistical Signal Processing (SSP), pp. 209212.

[9] Ristic, B., Gunatilaka, A., 2008. Information driven localisation of a radiological point source, Information Fusion 9(2), pp.317326.

[10] Ristic, B., Arulampalam, S. and Gordon, N., 2003. Beyond the Kalman filter: Particle filters for tracking applications. Artech house.

[11] Yee, E., 2007. Bayesian probabilistic approach for inverse source determination from limited and noisy chemical or biological sensor concentration measurements, Defense and Security Symposium, International Society for Optics and Pho- tonics, pp. 65540W65540W.

[12] Platt, N., DeRiggi, D., 2012. Comparative investigation of source term estimation algorithms using fusion field trial 2007 data: linear regression analysis. Int. J.Environ. Pollut. 48(14), pp. 13-21.

[13] Storwold, D., 2007. Detailed test plan for the fusing sensor information from observing networks (fusion) field trial 2007 (fft 07), US Army Dugway Proving Ground West Desert Test Center, Doc. WDTC-TP-07078 .

[14] Arulampalam, M.S., Maskell, S., Gordon, N. and Clapp, T., 2002. A tutorial on particle filters for online nonlinear/non-Gaussian Bayesian tracking. IEEE Transactions on signal processing, 50(2), pp.174-188.

[15] Li, T., Bolic, M. and Djuric, P. M, 2015. Resampling methods for particle filtering: classification, implementation, and strategies, IEEE Signal Processing Magazine, 32(3), pp. 70-86.

[16] Doucet, A., Godsill, S. and Andrieu, C., 2000. On Sequential Monte Carlo Sampling Methods for Bayesian Filtering, Statistics and Computing, 10(3), pp. 197-208.

[17] Hutchinson, M., Liu, C. and Chen, W.H., 2018. Information based mobile sensor planning for source term estimation of a non-continuous atmospheric release, in: International Conference on Robotics and $\mathrm{Au}-$ tomation (ICRA), pp. 1-6.

[18] Yee,E., 2017. Automated Computational Inference Engine for Bayesian Source Reconstruction: Application to Some Detections/Non-detections Made in the CTBT International Monitoring System, Applied Mathematical Sciences, 11(32), 1581-1618.

[19] Senocak, I., Hengartner, N.W., Short, M.B. and Daniel, W.B., 2008 Stochastic event reconstruction of atmospheric contaminant dispersion using Bayesian inference, Atmospheric Environment, 42(33), 77187727. 